\title{
INEQUALITIES FOR THE FROBENIUS NORM
}

\section{YANG PENG}

Abstract. In this note, we present a refinement of Heinz inequality for the Frobenius norm and discuss the relationship between our result and some existing inequalities

Mathematics subject classification (2010): 15A15, 15A42, 15A60.

Keywords and phrases: Positive semidefinite matrix, matrix means, Frobenius norm.

\section{REFERENCES}

[1] R. Bhatia, Matrix Analysis, Springer-Verlag, New York, 1997.

[2] R. Bhatia, C. DAVIS, More matrix forms of the arithmetic-geometric mean inequality, SIAM J. Matrix Anal. Appl. 14 (1993), 132-136.

[3] X. HU, Some inequalities for unitarily invariant norms, J. Math. Inequal. 6 (2012), 615-623.

[4] F. Kittaneh, Y. MAnASRAh, Improved Young and Heinz inequalities for matrices, J. Math. Anal. Appl. 361 (2010), 262-269.

[5] L. Zou, Inequalities related to Heinz and Heron means, J. Math. Inequal. 7 (2013), 389-397.

[6] L. Zou, C. HE, On some inequalities for unitarily invariant norms and singular values, Linear Algebra Appl. 436 (2012), 3354-3361. 